\title{
Comparison of gene editing versus conventional breeding to introgress the POLLED allele into the US dairy cattle population
}

\author{
M. L. Mueller, ${ }^{1}$ J. B. Cole, ${ }^{2}$ T. S. Sonstegard, ${ }^{3}$ and A. L. Van Eenennaam ${ }^{1 *}$ \\ ${ }^{1}$ Department of Animal Science, University of California, Davis 95616 \\ ${ }^{2}$ USDA, Agricultural Research Service, Animal Genomics and Improvement Laboratory, Beltsville, MD 20705-2350 \\ ${ }^{3}$ Acceligen, St. Paul, MN 55104
}

\section{ABSTRACT}

Disbudding and dehorning are commonly used cattle management practices to protect animals and humans from injury. They are unpleasant, costly processes subject to increased public scrutiny as an animal welfare issue. Horns are a recessively inherited trait, so one option to eliminate dehorning is to breed for polled (hornlessness). However, due to the low genetic merit and scarcity of polled dairy sires, this approach has not been widely adopted. In March 2018, only 3 Holstein and 0 Jersey active homozygous polled sires were registered with the National Association of Animal Breeders. Alternatively, gene editing to produce high-geneticmerit polled sires has been proposed. To further explore this concept, introgression of the POLLED allele into both the US Holstein and Jersey cattle populations via conventional breeding or gene editing (top $1 \%$ of bulls/year) was simulated for 3 polled mating schemes and compared with baseline selection on lifetime net merit (NM\$) alone, over the course of $20 \mathrm{yr}$. Scenarios were replicated 10 times and the changes in HORNED allele frequency, inbreeding, genetic gain $(\mathrm{NM} \$)$, and number of unique sires used were calculated. Gene editing decreased the frequency of the HORNED allele to $<0.1$ after $20 \mathrm{yr}$, which was as fast or faster than conventional breeding for both breeds. In the mating scheme that required the use of only existing homozygous polled sires, inbreeding reached 17\% (Holstein) and $14 \%$ (Jersey), compared with less than $7 \%$ in the baseline scenarios. However, gene editing in the same mating scheme resulted in significantly less inbreeding, 9\% (Holstein) and 8\% (Jersey). Also, gene editing resulted in significantly higher $\mathrm{NM} \$$ after $20 \mathrm{yr}$ compared with conventional breeding for both breeds. Additionally, the gene editing scenarios of both breeds used a significantly greater number of unique sires compared

Received October 23, 2018.

Accepted January 21, 2019.

*Corresponding author: alvaneenennaam@ucdavis.edu with either the conventional breeding or baseline scenarios. Overall, our simulations show that, given the current genetic merit of horned and polled dairy sires, the use of conventional breeding methods to decrease the frequency of the HORNED allele will increase inbreeding and slow genetic improvement. Furthermore, this study demonstrates how gene editing could be used to rapidly decrease the frequency of the HORNED allele in US dairy cattle populations while maintaining the rate of genetic gain, constraining inbreeding to acceptable levels, and simultaneously addressing an emerging animal welfare concern.

Key words: polled, gene editing, Holstein, Jersey, simulation

\section{INTRODUCTION}

Improved food animal welfare is of growing interest to consumers worldwide (Rutgers, 2003). Particularly, the practices of stopping horn bud growth (disbudding) or removing attached horns (dehorning) from cattle have garnered attention as animal welfare issues (Ventura et al., 2015). Horn removal is done to improve safety of human handlers, decrease risk of injury to other cattle through aggressive behaviors, and reduce the incidence of carcass wastage due to bruising (AVMA, 2014).

In the United States, $94 \%$ of dairy cattle producers report routine dehorning (20\%) or disbudding (80\%) (USDA, 2014). Calves less than 8 wk old are disbudded, which destroys or removes the horn-producing cells, stopping horn development before skull attachment. Older calves are dehorned, effectively amputating the fixed horn from the skull. Both procedures cause behavioral, physiologic, and neuroendocrine changes, indicating stressful or painful responses (Stafford and Mellor, 2011).

Alternatively, genetic selection can be used to breed for polled (hornless) cattle. Horns are inherited as an autosomal recessive trait (Long and Gregory, 1978). Three candidate POLLED mutations have been predicted in Bos taurus cattle on chromosome 1 (BTA1; Medugorac et al., 2012, 2017). One is an 80,128-bp 
duplication of Friesian origin $\left(\mathbf{P}_{\mathbf{F}}\right.$; Rothammer et al., 2014). The second is a simple allele of Celtic origin $\left(\mathbf{P}_{\mathbf{C}}\right)$ corresponding to a duplication of $212 \mathrm{bp}$ in place of a 10-bp deletion. Recently, a third allele, a complex 219-bp duplication-insertion $\left(\mathrm{P}_{219 \mathrm{ID}}\right)$, and a 7-bp deletion and 6-bp insertion $\left(\mathrm{P}_{1 \mathrm{ID}}\right)$ were identified through admixed Mongolian yaks (Medugorac et al., 2017). The predominant POLLED mutation in Holsteins and Jerseys is $\mathrm{P}_{\mathrm{F}}$ (Medugorac et al., 2012).

Global animal health organizations strongly recommend breeding for polled cattle whenever possible (AVMA, 2014; OIE, 2018). However, the frequency of the POLLED allele remains very low in dairy breeds, $1.1 \%$ and $2.2 \%$ in US Holsteins and Jerseys, respectively (Null et al., 2015). In March 2018, only 3 of 3,084 Holstein and 0 of 520 Jersey sires registered with the National Association of Animal Breeders were active and homozygous polled (NAAB, 2018). Additionally, polled dairy sires are on average $\$ 100$ lower on lifetime net merit (NM\$) than horned dairy sires (Figure 1). However, it is important to note that horns do not have a cause and effect relationship with genetic merit; rather they came along as genetic hitchhikers when selecting for elite dairy genetics and often the observed differences between polled and horned cattle have been confounded by the intensities of selection placed on different traits (Goonewardene et al., 1999a,b; Windig et al., 2015). Thus, even crossbreeding within dairy breeds to increase the frequency of the POLLED allele would have to occur gradually to prevent major economic effects on genetic diversity and production (Tan et al., 2012).

Gene editing to achieve an intraspecies $\mathrm{P}_{\mathrm{C}}$ allele introgression (212-bp duplication replacing a 10-bp sequence on chromosome 1) has been achieved (Carlson et al., 2016). Simulation studies have demonstrated that the use of gene editing is the most effective way to decrease the frequency of recessive alleles (e.g., HORNED), while minimizing detrimental effects on inbreeding and genetic merit (Cole, 2017a; Bastiaansen et al., 2018). However, neither study modeled the attributes of the current US dairy population (i.e., proportion of polled sires available or differences in genetic merit).

The objective of this study was to model the current US Holstein and Jersey dairy cattle populations and simulate the introgression of the POLLED allele by conventional breeding compared with gene editing for 3 different polled mating schemes. The change in HORNED allele frequency, inbreeding, genetic gain $(\mathrm{NM} \$)$, and the number of unique service sires used was determined for both introgression methods, and contrasted to conventional selection on $\mathrm{NM} \$$ alone, for a total of 7 scenarios analyzed.

\section{MATERIALS AND METHODS}

Computer simulation using geneedit.py (Cole, 2017b) was used to compare the introgression of the POLLED allele into the US dairy cattle population via conventional breeding versus gene editing. The program simulates gene editing applied to a dairy cattle population as an extension of Cole's (2015) program to manage multiple recessives and was modified to include additional parameters for selection of polled sires.

\section{Base Population}

The base population for both breeds was 35,000 cows distributed over 200 herds and 350 bulls. These animals were assigned a birth year from -4 to 0 (cows) or -9 to 0 (bulls) by sampling from a uniform distribution. True breeding values for $\mathrm{NM} \$$ were determined by randomly sampling from a normal distribution, with a mean of $\$ 0$ for cows and $\$ 300$ for bulls, and genetic SD of $\$ 200$ for both (Cole, 2015). The proportion of polled bulls in the Holstein base population was set to $2.1 \%$ heterozygous and $0.5 \%$ homozygous. These polled Holstein bulls averaged $0.9 \mathrm{SD}$ and 1.5 SD lower $\mathrm{NM} \$$, respectively, than horned bulls (NAAB, 2017). In the Jersey simulations, the proportion of base population polled bulls was $5.4 \%$ heterozygous and $1.5 \%$ homozygous. These polled Jersey bulls averaged $0.5 \mathrm{SD}$ and $1.3 \mathrm{SD}$ lower $\mathrm{NM} \$$, respectively, than horned bulls (NAAB, 2018). The frequency of the HORNED allele in the whole

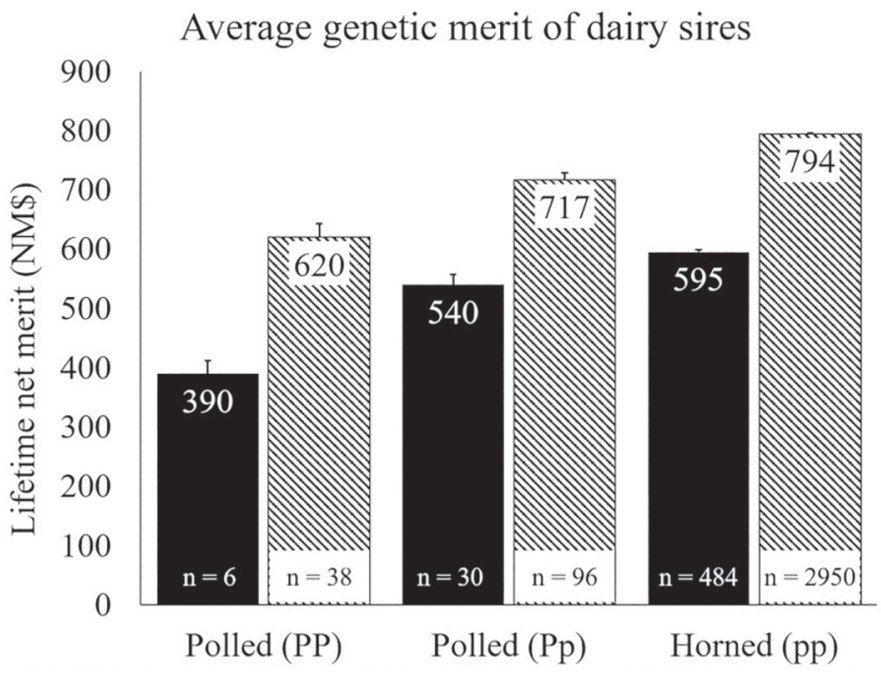

Figure 1. Average lifetime net merit (NM $\$$ ) of the top $50 \%$ polled and horned Holstein (black and white hatched bars) and Jersey (solid black bars) sires and the total number of each genotype and breed registered with the National Association of Animal Breeders under active, foreign, genomic, or limited status in March 2018 (NAAB, 2018). Error bars represent SEM. 
base population was 0.993 for Holstein and 0.978 for Jersey (Null et al., 2015). An animal's horned status was determined by randomly sampling sire and dam alleles using the corresponding breed HORNED allele frequencies. The population increased to a maximum of 500 bulls and 100,000 cows over $20 \mathrm{yr}$, with overlapping generations.

\section{Descendants}

The true breeding values (TBV) for new calves were created by taking the parent average and adding a Mendelian sampling term $(\mathrm{MS}): \mathrm{TBV}_{\text {calf }}=0.5\left(\mathrm{TBV}_{\text {sire }}\right.$ $\left.+\mathrm{TBV}_{\mathrm{dam}}\right)+\mathrm{MS}$, where $\mathrm{TBV}_{\text {calf }}, \mathrm{TBV}_{\text {sire, }}$ and $\mathrm{TBV}_{\text {dam }}$ are the TBV of the calf, its sire, and its dam, respectively. The Mendelian sampling term was drawn from a normal distribution with a mean of 0 and a variance of $\frac{1}{2}\left[1-\frac{1}{2}\left(f_{S}+f_{D}\right)\right] \sigma_{a}^{2}$, where $f_{S}$ and $f_{D}$ are the coefficients of inbreeding of the sire and dam, respectively, and $\sigma_{a}^{2}$ is the additive genetic variance of $\mathrm{NM} \$$ (Cole, 2015). Sex was assigned randomly with a 50:50 sex ratio. Calves were born in the same herd as their dams, and cows did not move between herds. To determine a new calf's horned status, one allele was sampled at random from each parent and used to construct the progeny genotype. Recessive genotypes were simulated without error, and it was only necessary to simulate genotypes for recessive alleles because pedigrees were assumed to be free of errors. Allele frequencies were updated each year by counting alleles (Cole, 2015).

\section{Mating Scheme Designs}

Four mating schemes, 1 baseline (A) and 3 polled (B-D) were modeled (Table 1). To establish a baseline and model current practice, scheme A used $\mathrm{NM} \$$ as the sole sire selection criterion and each bull was limited to 5,000 matings per year (5\% of the total offspring/ year) to prevent the overuse of any one sire (Cole, 2015). Additionally, 3 polled mating schemes (B-D) were modeled where polled sires were preferentially or obligatorily selected over horned sires, regardless of the difference in $\mathrm{NM} \$$. In scheme B, polled bulls (both heterozygous and homozygous) were preferentially selected and mated with a mating limit (5,000 matings per year) for each bull. If the maximum number of matings was reached for all polled bulls before all cows were bred, horned sires were used until all of the cows were allocated mates. In scheme $\mathrm{C}$, only homozygous polled bulls were preferentially selected until the mating limit (5,000 matings per year) was reached, and then both heterozygous polled and horned sires were used for the remaining matings when necessary. In contrast to these 2 polled preference scenarios, in scheme $\mathrm{D}$ only homozygous polled bulls could be used and no annual mating limit was set for each bull. This scheme models what might be expected if producers are prohibited from using genetics that results in horned offspring.

\section{Mate Allocation}

All scenarios used the modified Pryce (pryce_r) scheme to allocate bulls to cows (Cole, 2015). The pryce_r scheme penalizes the parent average $\mathrm{NM} \$$ to account for matings that would result in increased inbreeding (Pryce et al., 2012) and the economic costs of horned and carrier offspring (Cole, 2015; Mueller et al., 2018a,b). The inbreeding depression penalty associated with a $1 \%$ increase in inbreeding was calculated to be $\$ 25$ (Cole, 2015). Inbreeding was estimated without error based on pedigree relationships (Aguilar and Misztal, 2008) using the program INBUPGF90 (Aguilar and Misztal, 2012). The average expected cost of disbudding is estimated to range from $\$ 6$ to $\$ 25$ per animal, depending on the method used and the cost of labor, equipment, and veterinary treatments for infections/blood loss (Thompson et al., 2017). Therefore, to additionally account for breeders' preferences and premium marketing opportunities, horned and carrier calves were penalized $\$ 40$ and $\$ 20$, respectively, in all schemes.

\section{Gene Editing}

The 3 polled mating schemes (B-D) described above were also simulated with the addition of gene editing for polled, which, when combined with the baseline section on $\mathrm{NM} \$$ alone, made a total of 7 scenarios. In these scenarios (Edit-B, Edit-C, and Edit-D), gene editing was modeled as an added step to the production system proposed by Kasinathan et al. (2015), which combines the use of advanced reproductive technologies and somatic cell nuclear transfer cloning with embryo transfer to produce elite sires. In the Edit- scenarios, the bulls were sorted on $\mathrm{NM} \$$ in descending order, and the top $1 \%$ of heterozygous polled and horned bulls were cloned and then edited to be homozygous polled.

\section{Data Analysis and Visualization}

Each scenario was replicated 10 times using a different seed for the random number generator. The actual values used were saved to an output file so that results could be replicated if necessary. The change in $H O R N E D$ allele frequency, inbreeding, $\mathrm{NM} \$$, and the 


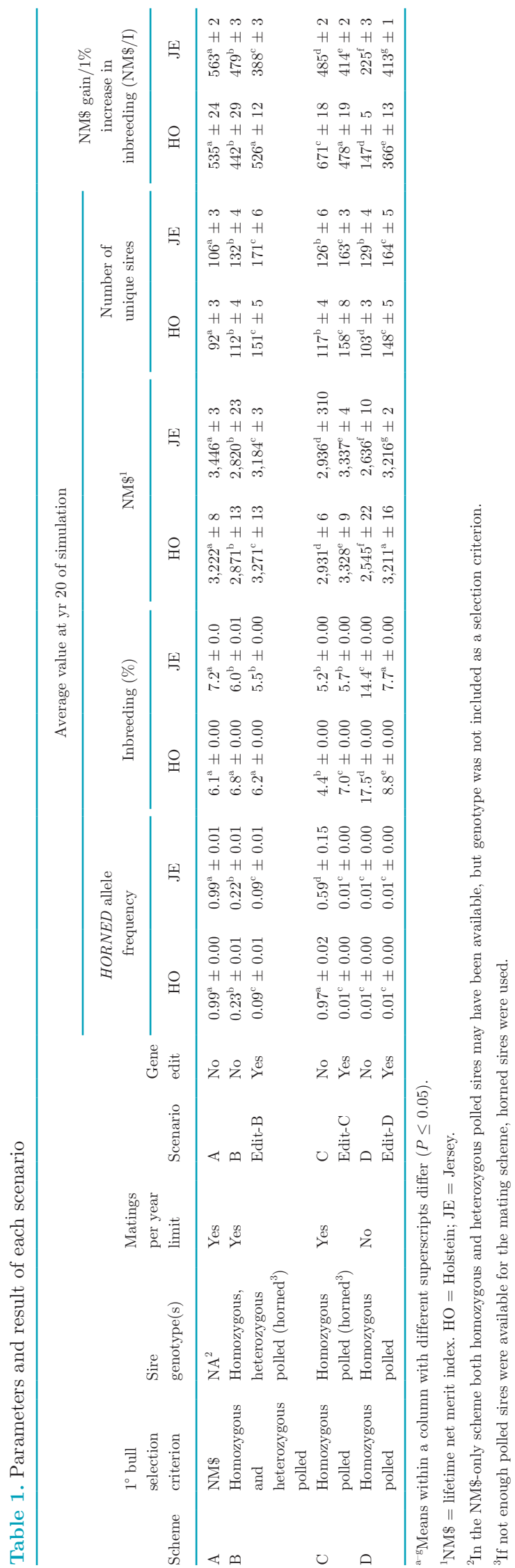

number of unique service sires used was compared between (1) conventional breeding (B-D) versus gene editing (Edit-B, Edit-C, and Edit-D) scenarios within a mating scheme and (2) each scenario versus the baseline (A). Significance of the change in HORNED allele frequency, inbreeding levels, and the number of sires used was determined using 2-tailed, unpaired student $t$-tests. Linear regressions of true breeding value for $\mathrm{NM}$ \$ on birth year were used to determine significance of change in the rate of genetic gain $(\mathrm{NM} \$)$. $P$-values of $\leq 0.05$ were considered to be significantly different for all analyses. The results (Figure 2-4) presented are the means of the 10 replicates for each scenario and the variation between the replicates is represented by standard error of the mean bars (Figure 2). Additionally, the efficiency of each scenario was calculated in terms of rate of genetic gain $(\mathrm{NM} \$)$ achieved per $1 \%$ of genetic variance lost $(\mathbf{N M} \mathbf{S} / \mathbf{I}$; Table 1).

\section{RESULTS AND DISCUSSION}

\section{HORNED Allele Frequency}

The baseline results (A) support findings by Cole (2015), which concluded that a horned economic penalty $(\$ 40)$ in the selection index alone is not effective at decreasing the HORNED allele frequency in the US dairy population (Figure 2a-b). In this scenario, the $P O L L E D$ allele frequency was so low that carriers were unlikely to be one of the top-ranked bulls based on adjusted $\mathrm{NM} \$$, so almost all sires used were horned (Figure 3). In contrast, when polled sires were preferentially selected in scheme $\mathrm{B}$, the HORNED allele frequency decreased significantly $(P \leq 0.01)$ compared with scheme A for both breeds when modeling conventional breeding. The addition of gene editing (Edit-B) resulted in a significantly faster $(P \leq 0.01)$ decrease in HORNED allele frequency after $20 \mathrm{yr}$ (Holstein and Jersey $=0.09)$ as compared with conventional breeding $($ Holstein $=0.23$; Jersey $=0.22)$.

Scheme $\mathrm{C}$ resulted in the largest difference between breeds in HORNED allele frequency after $20 \mathrm{yr}$. In Holstein, after $20 \mathrm{yr}$ of conventional breeding the HORNED allele frequency was 0.97 , which was not significantly different $(P=0.71)$ than A, whereas in Jersey the frequency was 0.59 , which was significantly lower $(P \leq$ 0.01 ) than A. In this mating scheme, only homozygous polled sires were preferentially selected and the sire mating limit was in place. This resulted in $\sim 1$ homozygous polled sire and several horned sires being used in both breeds in yr 1 . However, the frequency of the $P O L L E D$ allele was higher in Jersey, so by chance a few $(\sim 3-4)$ heterozygous polled sires were also used based only on their $\mathrm{NM} \$$, whereas only $\sim 1$ Holstein heterozy- 
gous polled sire was used (Figure 3). These differences in yr 1 sire choices resulted in more Jersey polled sires being available in subsequent years as compared with the Holstein scenario. However, the Jersey results were quite variable $(\mathrm{SEM}=0.15)$ between replicates, driven by the polled sire availability in yr 1 . In contrast, using gene editing (Edit-C) rapidly increased the number of high NM\$ homozygous polled sires in both breeds, so no horned sires were used after yr 2. As a result, $H O R N E D$ allele frequency in the Edit-C scenarios of both breeds decreased significantly faster $(P \leq 0.01)$ than conventional breeding in the same scheme (Figure $2 \mathrm{a}, 2 \mathrm{~b})$.

Only homozygous polled sires were used in scheme D, so both conventional breeding and gene editing resulted in the same $(P>0.10)$ rapid change in HORNED allele frequency in both Holstein and Jersey. Overall, gene editing decreased HORNED allele frequency as fast, or faster, than conventional breeding in each mating scheme for both breeds (Figure 2a, 2b).

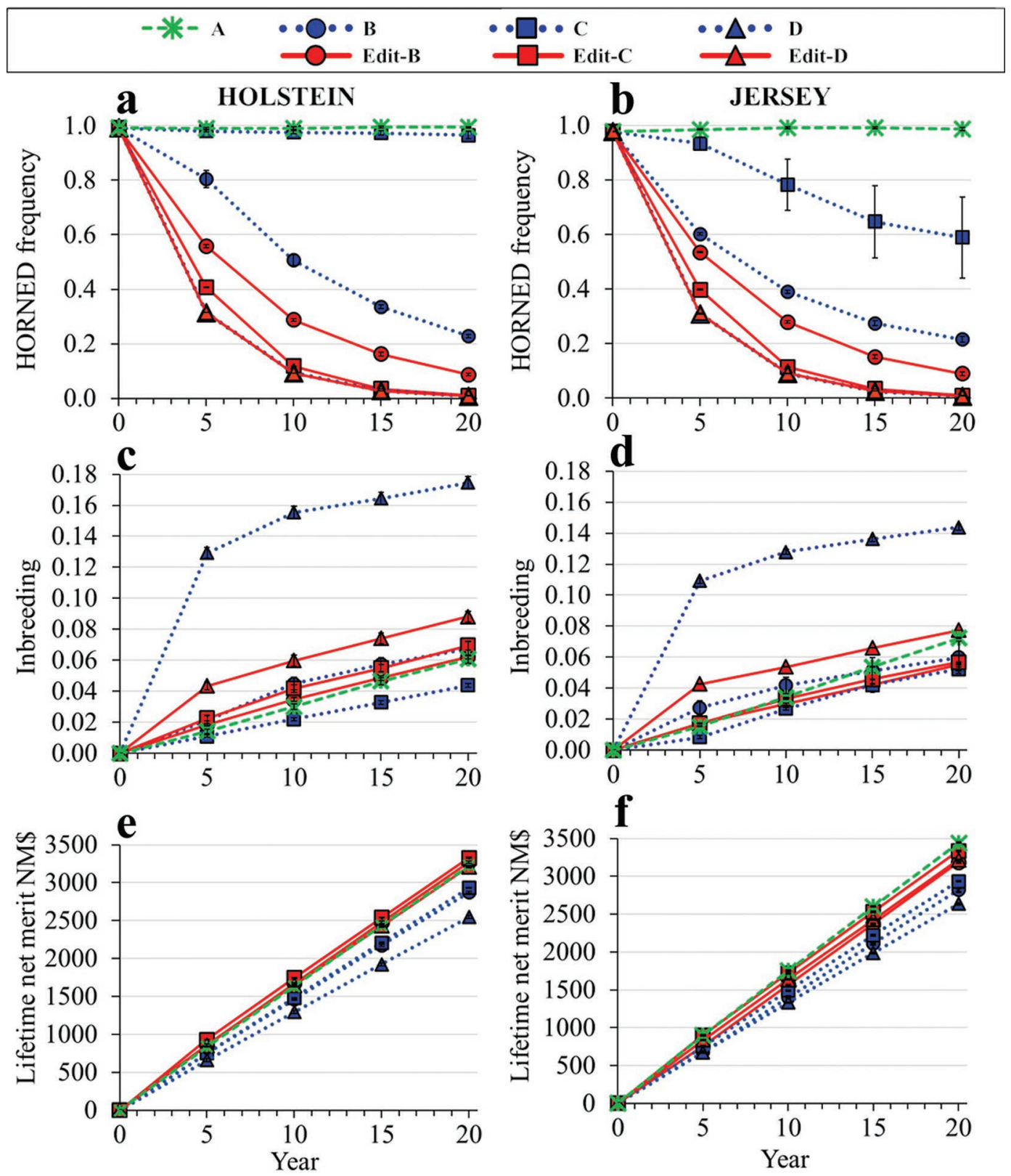

Figure 2. Effect of each mating scenario on ( $\mathrm{a}, \mathrm{b})$ HORNED allele frequency, (b, c) inbreeding, and (e, f) genetic merit for Holstein (a, c, d) and Jersey (b, d, f). Scenario A is a dashed line, polled conventional breeding scenarios (B, C, D) are dotted lines, and polled gene editing scenarios (Edit-B, Edit-C, Edit-D) are solid lines. Error bars represent SEM. 


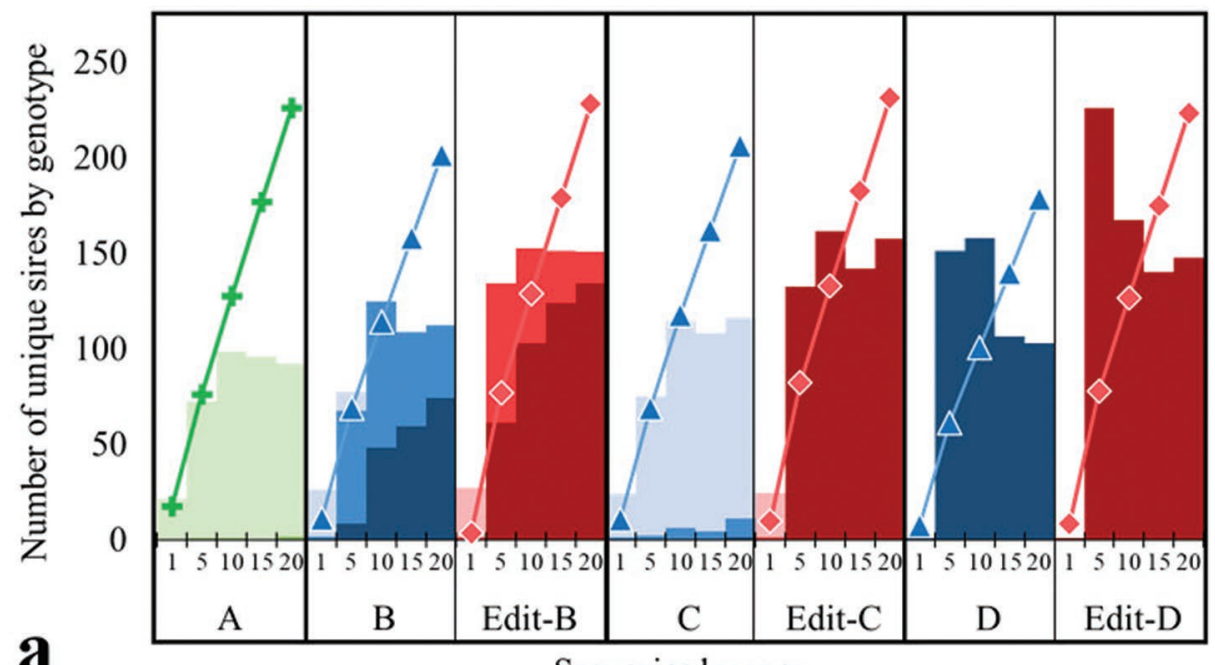

4000

3500

3000

2500

2000

1500

1000

500

0

$\mathbf{a}$

Scenarios by year

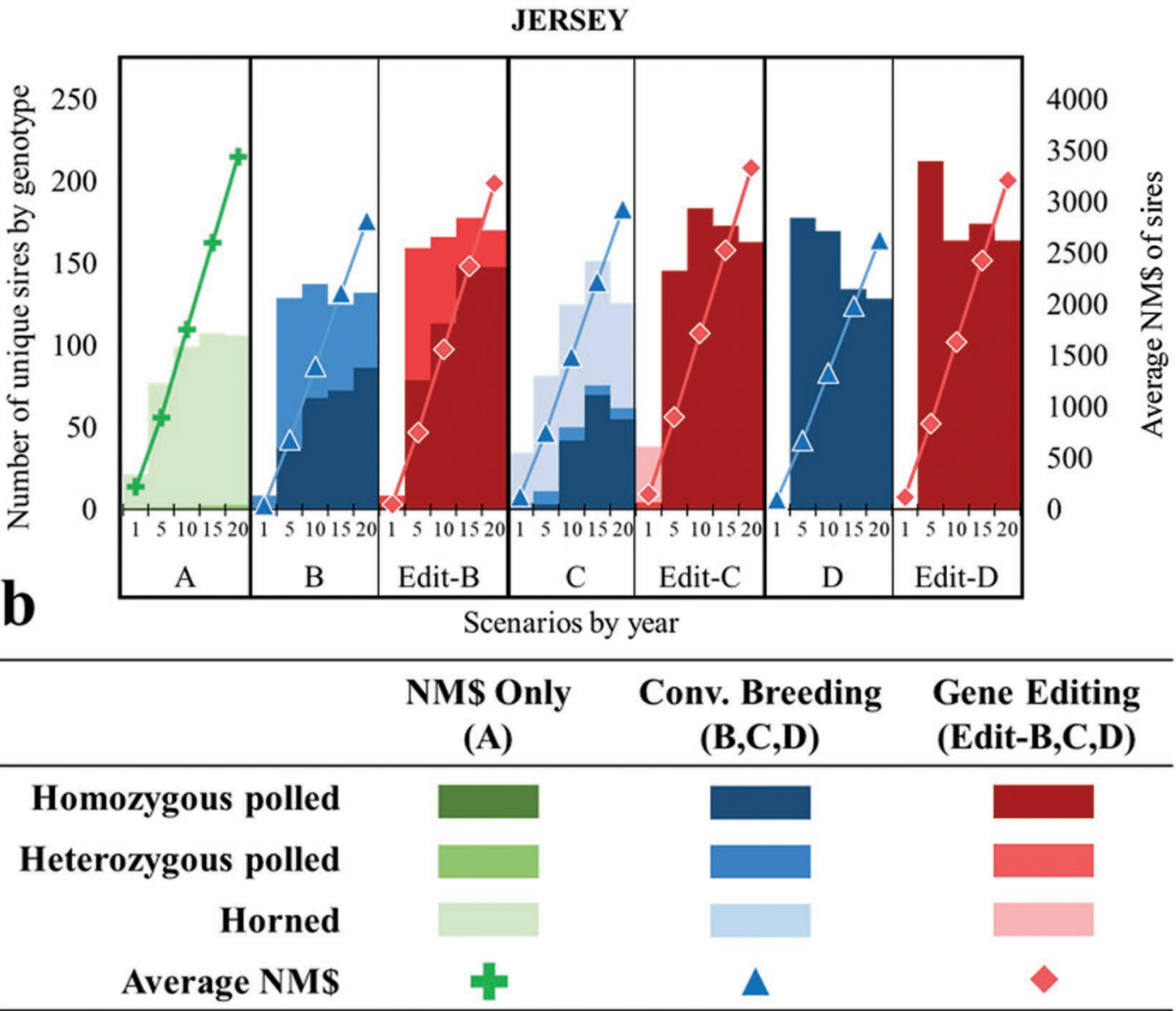

Figure 3. Effect of each mating scenario on the number of unique sires used in yr 1, 5, 10, 15, and 20 by genotype on the primary y-axis and the average lifetime net merit $(\mathrm{NM} \$$ ) of all sires per scenario on the secondary y-axis for (a) Holsteins and (b) Jerseys. Genotypes are represented by a color scale from darkest (homozygous polled) to lightest (horned). The average $\mathrm{NM} \$$ of the sires for scenario A are represented by plus signs, polled conventional (Conv.) breeding scenarios (B, C, D) by blue triangles, and polled gene editing scenarios (Edit-B, Edit-C, and Edit-D) by red diamonds. 


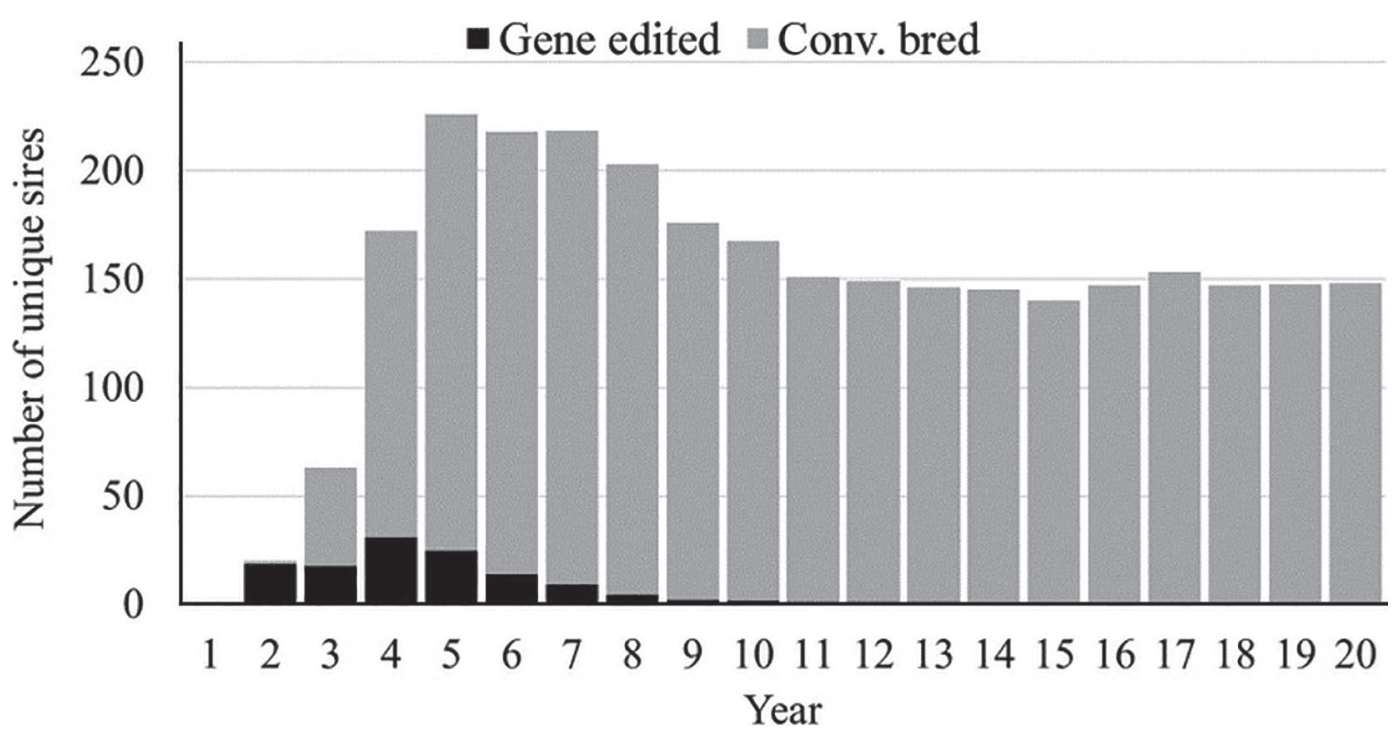

Figure 4. The number of unique gene-edited and conventionally (Conv.) bred sires used per year in the Edit-D Holstein scenario. Gene-edited sires are represented by black bars, and conventionally bred sires are represented by gray bars.

\section{Inbreeding}

For all scenarios, inbreeding was estimated without error based on pedigree relationships (Aguilar and Misztal, 2008). After 20 yr of scheme A, inbreeding reached $\sim 6.6 \%$ for both breeds, which is within industry norms. In Jersey, the polled preference mating schemes (B and C) resulted in lower inbreeding than A (Figure 2c). In contrast, preferentially selecting for polled in Holstein resulted in higher inbreeding than A in all scenarios except for $\mathrm{C}$ with conventional breeding (Figure 2d). It should be noted that in this Holstein C scenario, conventional breeding was not effective for decreasing the HORNED allele frequency, whereas this scenario in Jersey did significantly decrease $(P \leq$ $0.01)$ the HORNED allele frequency compared with A. In both breeds, the polled preference mating schemes used an additional selection criterion in the breeding objective, so herds used a wider variety of sires than A (Figure 3). However, due to the difference in POLLED allele frequency in the base populations, fewer polled Holstein sires were available compared with the Jersey population in the early simulation years, which resulted in higher inbreeding levels in Holstein. Both simulations assumed that all base population animals were initially unrelated, which is unlikely in a commercial setting.

In both breeds, scheme B used a mixture of sire genotypes as a result of preferred selection on both heterozygous and homozygous polled sires and having the mating limit in place (Figure 3 ). The addition of gene editing to this mating scheme (Edit-B) resulted in similar inbreeding levels compared with B scenarios of both breeds after $20 \mathrm{yr}$. In contrast, including gene editing in the Edit-C scheme increased inbreeding rates in both breeds compared with conventional breeding, due to using only homozygous polled sires after yr 2 . However, in Jerseys the Edit-C inbreeding level at yr 20 was still below the inbreeding level of scenario A (Figure 2d).

Scheme D limited herds to the use of only homozygous polled sires. Inbreeding in the conventional breeding scenarios was significantly higher $(P \leq 0.01)$ than the A scenarios of both breeds. The Edit-D scenarios of both breeds resulted in significantly less $(P \leq 0.01)$ inbreeding than conventional breeding for this mating scheme. In fact, inbreeding in the Edit-D Jersey scenario was not significantly different $(P=0.09)$ from the A scenario.

\section{Genetic Gain (NM\$)}

Selection of polled sires in the conventional breeding scenarios resulted in significantly slower $(P \leq 0.01)$ rates of genetic gain $(\mathrm{NM} \$)$ compared with the A scenarios of both breeds (Figure 2e, 2f), which is consistent with previous findings by Spurlock et al. (2014). Based on 2018 NAAB sire records, the currently available naturally polled sires of both breeds are, on average, $\$ 100$ lower $\mathrm{NM} \$$ than their horned counterparts (NAAB, 2018). Therefore, genetic gain is slowed when herds primarily use sires based on polled status rather than $\mathrm{NM} \$$ alone.

Of the 3 polled conventional breeding scenarios (BD), scenario C resulted in the fastest genetic gain for 
both breeds, although it was still significantly slower $(P$ $\leq 0.01$ ) than the A scenarios (Figure 2e, 2f). Recall that in this mating scheme only homozygous polled sires are preferentially selected, and the mating limit is in place so horned and heterozygous polled sires were also selected based only on their NM\$ (Figure 3). However, in both breeds the $\mathrm{C}$ conventional breeding scenarios resulted in the smallest decrease in HORNED allele frequency after $20 \mathrm{yr}$ (Holstein $=0.97$; Jersey $=0.59$ ) of all the polled mating scenarios (Figure 2a, 2b). This $\mathrm{C}$ scenario demonstrates the trade-offs between the ability to rapidly decrease the HORNED allele frequency and maintain genetic gain when only using conventional breeding methods. On the other hand, including gene editing in this same mating scheme for both breeds (Edit-C) resulted in the greatest genetic gain at yr 20 $(>\$ 3,300)$ of all the polled mating schemes $(\mathrm{B}-\mathrm{D})$ while still decreasing the HORNED allele frequency to $\leq 0.01$ after $20 \mathrm{yr}$.

For both breeds, the greatest difference in $\mathrm{NM} \$$ ( $>$ \$550) within a mating scheme after 20 yr was observed between the $\mathrm{D}$ conventional breeding versus gene editing (Edit-D) scenarios (Figure 2e, 2f). In this mating scheme, only homozygous polled sires were used with no mating limit. In yr $1, \sim 1$ homozygous polled sire was used for both the conventional breeding and gene editing scenarios with approximately the same $\mathrm{NM} \$$. However, by year 5 the average $\mathrm{NM} \$$ of the sires used in the Edit-D scenarios were $\$ 150+$ greater than the sires used in the conventional breeding scenarios (Figure 3). In the conventional breeding D scenarios, the homozygous polled sires continually lagged behind horned sires in terms of $\mathrm{NM} \$$, so genetic gain was slowed. In contrast, gene editing (Edit-D) resulted in the top ranked NM $\$$ bulls also being homozygous polled in just a few years, so rates of genetic gain could be maintained while still rapidly decreasing the HORNED allele frequency.

Results for the B mating scheme were intermediate to the $\mathrm{C}$ and $\mathrm{D}$ schemes discussed above. Overall, gene editing resulted in significantly faster $(P \leq 0.01)$ rates of genetic gain than conventional breeding in all polled mating schemes of both Holsteins and Jerseys (Figure $2 \mathrm{e}, 2 \mathrm{f})$.

\section{Number of Unique Sires Used}

Scenario A of both breeds used the lowest number of unique sires at yr 20 (Figure 3) due to only including one selection criterion $(\mathrm{NM} \$)$ in the breeding objective. Therefore, in this mating scheme polled sires were only used if they also were top-ranked in $\mathrm{NM} \$$. As a result, in the Holstein population, which had the lower base population POLLED allele frequency, 0 polled sires were used in yr 1 and after 20 yr 0 homozygous polled sires and only 2 heterozygous polled sires were used (Figure $3 a$ ). The Jersey A scenario used 0.3 homozygous polled and 1 heterozygous polled in addition to the 21 horned sires in yr 1 and after $20 \mathrm{yr}$ included 3 heterozygous polled sires (Figure $3 \mathrm{~b}$ ).

In yr 1 of mating scheme B, 24 horned and 2 heterozygous polled sires were used in the Holstein population (Figure 3a). In contrast, due to the higher base population POLLED allele frequency, enough polled Jersey sires were available, so no horned sires had to be used even in yr 1 (Figure 3b). In Holstein, including gene editing (Edit-B) resulted in horned sires only being used for the first $2 \mathrm{yr}$, whereas with conventional breeding horned sires continue to be used for $6 \mathrm{yr}$.

Similarly, in mating scheme $\mathrm{C}$ more polled Jersey sires were available to use in yr 1 compared with Holsteins. In this mating scheme, only homozygous polled sires were preferentially selected, so heterozygous polled sires were only used if selected based on their adjusted NM\$. As a result, in the conventional breeding scenarios most horned sires continued to be used in both breeds for all 20 yr. However, 55 homozygous polled Jersey sires were used at yr 20 compared with only 1 homozygous polled Holstein sire. In both breeds, including gene editing (Edit-C) resulted in only homozygous polled sires being used after yr 3 (Figure 3 ).

In mating scheme D of both breeds, only 1 homozygous polled sire was available and used in yr 1 of both the conventional breeding and gene editing scenarios. In yr 2 of the conventional breeding scenarios, $\sim 2$ homozygous polled sires were available and used. In contrast, by yr 2 of the gene editing scenarios (Edit-D) over 10fold more homozygous polled sires were available and used (Figure 3). The largest number $(>200)$ of unique sires used in 1 yr was observed in yr 5 of the Edit-D scenario. Due to the mate allocation process penalizing increased inbreeding, even though homozygous polled sires were allowed unlimited matings, a large number of sires were used instead to manage the steep inbreeding increase from yr 1 when only 1 homozygous polled sire was available and mated to all cows. Conventional breeding took several years to produce many homozygous polled sires, but gene editing allowed for the rapid production of multiple homozygous polled sires in just a few years.

Overall, the gene editing scenarios of both breeds used a significantly greater $(P \leq 0.01)$ number of unique sires as compared with either the conventional breeding or A scenarios. Additionally, after only 2 yr of each 
of the gene editing scenarios, enough polled sires were available to remain within the mating limit for each sire without using any horned sires (Figure 3).

Although significantly more unique sires were used in the gene editing scenarios, after yr 2 the majority of service sires themselves have not been gene edited; rather they were the offspring of gene-edited sires from the previous years (Figure 4 ). At $\sim 15$ yr, no gene-edited sires were used because all of the highest-ranking NM\$ bulls were already homozygous polled based on inheritance from their parents, so gene editing for this trait was no longer required.

\section{Scenarios}

Recently, Thompson et al. (2017) conducted a cost-benefit analysis of traditional disbudding versus breeding for polled animals. They estimated the cost for disbudding, with the addition of treatment probability and costs included, to range from $\$ 6$ to $\$ 25$ per head, with a mean economic cost around $\$ 12$ to $\$ 13$ per head. Additionally, they estimated the expected cost of incorporating polled genetics to range from $\$ 0$ to $\$ 26$ per head, depending on the additional cost, or premium, associated with polled genetics. Combined, these estimates indicate that on average, producers could spend up to $\$ 5.95$ per head and $\$ 11.90$ per head more for heterozygous and homozygous polled genetics, respectively, compared with conventional horned genetics. However, this analysis ignored the difference in genetic merit and relatedness of polled sires compared with horned sires.

Factoring in the difference in genetic merit of currently available polled dairy sires, Spurlock et al. (2014) compared several conventional breeding strategies for incorporating polled genetics at the individual herd level for $10 \mathrm{yr}$ and estimated the effect of each strategy on the overall genetic merit of the herd. Although a $100 \%$ polled calf crop could be achieved by using only homozygous polled bulls, they demonstrated that this is not a cost-effective strategy. After $10 \mathrm{yr}$ of using only homozygous polled bulls each cow earned $\$ 252$ less in lifetime earnings than a herd using horned sires. Spurlock et al. (2014) highlighted the trade-offs that dairy producers must consider when evaluating the use of currently available polled genetics in their herds. Similar situations were reported in the Netherlands (Windig et al., 2015) and Germany (Scheper et al., 2016).

Increasing the frequency of the POLLED allele in the US dairy population through conventional breeding has several trade-offs. Spurlock et al. (2014) demonstrated these trade-offs on an individual herd basis, whereas our study evaluates the trade-offs from a population level. The scenarios were developed to represent potential situations that might arise if no, small, or drastic (i.e., prohibited production of horned calves) market pressure is placed on the US dairy industry to eliminate dehorning.

The optimal conventional breeding scenario in this study for both breeds was B, based on the criteria of an acceptable rate of $\mathrm{NM} \$$ improvement per $1 \%$ increase in inbreeding $(\mathrm{NM} \$ / \mathrm{I}$ : Holstein $=442$; Jersey $=$ 479 ) and decreasing the HORNED allele frequency to $<0.25$ by yr 20 . Scenario B preferentially selected and mated both heterozygous and homozygous polled sires with the mating limit in place, so horned sires could also be used. This polled preferential mating resulted in a significant decrease in HORNED allele frequency, whereas the mating limit constrained inbreeding and maintained genetic gain to baseline levels.

Scheme D models a case where consumer and market expectations force the dairy industry to eliminate dehorning immediately, thereby requiring the exclusive use of homozygous polled sires. The rate of NM\$ increase per $1 \%$ increase in inbreeding was significantly lower for the conventional breeding D scenario $(\mathrm{NM} \$ / \mathrm{I}$ : Holstein $=147$; Jersey $=225)$ as compared with all others (Table 1), because this scenario forced the use of related polled homozygous sires irrespective of their $\mathrm{NM} \$$ or relationship to each other. If this scenario were to play out, it would have very deleterious effects on dairy cattle genetic improvement programs. In this case, gene editing (Edit-D) could provide an alternative approach to avoid long-term detrimental effects on the inbreeding level and genetic merit of the US dairy industry as it allowed for an intermediate rate of $\mathrm{NM} \$$ increase per $1 \%$ increase in inbreeding (NM\$/I: Holstein $=366$; Jersey $=413)$, while still achieving the goal of reducing the HORNED allele frequency to $<0.01$ by yr 20 .

Producing high-genetic-merit polled sires through gene editing could mitigate many of the economic and inbreeding trade-offs associated with increasing the POLLED allele frequency. In Jerseys, the optimal gene editing scenario was Edit-C, based on the rate of $\mathrm{NM} \$$ improvement per $1 \%$ increase in inbreeding (NM\$/I: 414) of the scenarios that decreased HORNED allele frequency to $<0.01$ by yr 20 . This scenario preferentially selected and mated only homozygous polled sires with a sire mating limit in place, so both heterozygous polled and horned sires could also be used. Scheme C could represent a situation where some producers choose to only use homozygous polled sires, whereas others might continue to select sires based only on $\mathrm{NM} \$$. In the first few years when only a limited number of homozygous polled sires were available, having the mating limit in 
place kept inbreeding from increasing above baseline levels, and having the flexibility to continue to use both heterozygous and horned sires based on $\mathrm{NM} \$$ resulted in a rate of genetic gain similar to the baseline. In this Edit-C scenario, gene editing resulted in the rapid production of several high-genetic-merit homozygous polled sires, which ultimately resulted in the lowest HORNED allele frequency of 0.01 and highest genetic merit at $20 \mathrm{yr}$.

In Holsteins, a slightly better rate of $\mathrm{NM} \$$ improvement per $1 \%$ increase in inbreeding (Table 1) was obtained with the Edit-B scenario (NM\$/I: 526). However, the HORNED allele frequency was only reduced to 0.09 at 20 yr. For only a slight increase in inbreeding and therefore decrease in the rate of $\mathrm{NM} \$$ improvement per $1 \%$ increase in inbreeding $(\mathrm{NM} \$ / \mathrm{I}: 478)$, scenario Edit-C decreased the HORNED allele frequency to 0.01 at $20 \mathrm{yr}$ and resulted in the fastest rate of genetic gain. Ultimately the best scenario depends upon the weighting and priority placed upon each of the competing goals of increasing $\mathrm{NM} \$$, minimizing inbreeding, and reducing the HORNED allele frequency.

\section{Model Limitations}

There are some limitations of the simulation model used in this study. Most notably, pedigrees and genotypes are modeled without error, and it is assumed that the true QTL for each trait modeled is known. In reality, haplotype tests are used for many monogenic traits for which the true causal variant is unknown, and recombination can break down the association of the true causal variant with tag SNP in the haplotype, resulting in a loss of accuracy in prediction of true carrier status. The simulation also models only a small number of individual loci, rather than complete genotypes. This means that the inbreeding estimates, although estimated without error, are based only on pedigree relationships rather than shared DNA. It also means that more complex situations, such as the presence of 2 or more loci on the same chromosome, cannot be modeled properly. Finally, the selection objective included in the model is a total merit index, rather than individual traits, which precludes examination of interesting scenarios in which there is a favorable association of a Mendelian trait with one phenotype, and an unfavorable association with another.

The size of the simulated population in this study was 100,000 cows and 500 bulls, whereas the US dairy cow population is approximately 90 times larger (i.e., 9 million cows). Scaling up the number of gene-edited bulls/year to represent the number of bulls needed for the US dairy population would entail editing multiple AI sires. One limitation of the simulation modeled in this study was that the gene editing rate $(1 \%$ of bulls/year) remained constant throughout the whole simulation, even when gene editing for this trait was no longer required. Irrespective, for any of the gene editing scenarios, multiple unrelated high-merit bulls would need to be edited for each dairy breed to prevent an unacceptable increase in inbreeding.

\section{Regulatory Considerations}

The regulatory status of gene-edited animals will need to be clarified before it will be possible to introduce the POLLED allele into the top $1 \%$ of genetically elite bulls as was modeled in this study. The global status of gene-edited animals is uncertain and is a primary concern for further investment and development of gene-edited animals (Maxmen, 2017; Heller, 2018). Scientists have urged lawmakers and regulators to adopt a regulatory process based on novel product risks and benefits rather than the use of the gene editing in the breeding program (Wells, 2016; Carroll et al., 2016; Van Eenennaam, 2018). In March of 2018, the USDA announced that it would not require any additional pre-market regulations for gene-edited plants that could have been developed through traditional breeding techniques as long as they are not plant pests or developed using plant pests (Anonymous, 2018). Under this ruling genetic deletions, single base-pair substitutions, and the introgression of nucleotide sequences from related plants that could potentially have come about through cross-breeding, analogous to the $\mathrm{P}_{\mathrm{C}}$ intraspecies allele substitution, would all be outside the scope of additional pre-market USDA regulation (Anonymous, 2018). The USDA approach differs markedly from the Court of Justice of the European Union 2018 ruling, which states that gene-edited crops will be subject to the same regulations as conventional genetically modified organisms (Callaway, 2018).

When it comes to gene-edited food animals, the 2017 US Food and Drug Administration draft guidance declares that all animals whose genomes have been intentionally altered will be regulated as new animal drugs (FDA, 2017). As such, gene-edited polled animals would meet the definition of a veterinary drug. However, animals carrying the exact same POLLED allele sequence obtained by introgression or crossbreeding would not trigger regulatory oversight. In October 2018, the National Technical Biosafety Commission (CTNBio) in Brazil concluded that semen from an edited bull carrying the $\mathrm{P}_{\mathrm{C}}$ POLLED intraspecies allele substitution (Carlson et al., 2016) would not be considered a "genetically modified organism" under their regulatory schema (MCTIC, 2018). In the absence of regulatory harmony, breeders in some countries will 
have the ability use gene editing in agricultural breeding programs, whereas those in other countries will not, resulting in disparate breeder access to these tools, and ultimately the potential for trade disruptions.

\section{Consumer Acceptance}

Consumers may be more accepting of gene editing in food animals if the technology focus is on animal health and welfare rather than on productivity (Frewer et al., 2013; Van Eenennaam and Young, 2018). Additionally, consumers may have fewer objections to gene editing of animals that involve genetic variants already naturally occurring in the species (e.g., polled in cattle) rather than if the genetic variant is inserted from a different species (Croney et al., 2018; Schultz-Bergin, 2018). An ethical review of genetically modified and gene-edited animals concluded that gene editing for polled "may be one of the least controversial applications" (Eriksson et al., 2018).

\section{CONCLUSIONS}

Consumer concerns regarding improved animal welfare, specifically eliminating dehorning and disbudding, will likely continue to grow. Polled genetics are an option to replace dehorning. However, our simulations show that given the current availability and genetic merit of horned and polled dairy sires, conventional breeding to decrease the frequency of the HORNED allele will significantly increase inbreeding and slow genetic improvement. These resulting economic considerations are hindering the dairy industry's ability to address this animal welfare concern through currently available genetics. Although long-term progress can be made through conventional breeding, the negative effect on inbreeding and genetic merit would largely be ameliorated if gene editing could be used to introgress the $\mathrm{P}_{\mathrm{C}}$ allele into elite dairy genetics. Overall, this study demonstrates how gene editing could be used to rapidly decrease the frequency of the HORNED allele in US dairy cattle populations while maintaining the rate of genetic gain, constraining inbreeding to acceptable levels, and simultaneously addressing an emerging animal welfare concern.

\section{ACKNOWLEDGMENTS}

The authors acknowledge funding support for this work from the USDA National Institute of Food and Agriculture National Research Initiative Competitive Grant no. 2015-67015-23316 (T. S. Sonstegard, PD). Cole was supported by appropriated project 8042-31000-002-00-D, "Improving Dairy Animals by
Increasing Accuracy of Genomic Prediction, Evaluating New Traits, and Redefining Selection Goals," of the Agricultural Research Service of the United States Department of Agriculture. Mention of trade names or commercial products in this article is solely for the purpose of providing specific information and does not imply recommendation or endorsement by the US Department of Agriculture. The USDA is an equal opportunity provider and employer. The funders had no role in study design, data collection and analysis, decision to publish, or preparation of the manuscript.

\section{REFERENCES}

Aguilar, I., and I. Misztal. 2008. Technical Note: Recursive algorithm for inbreeding coefficients assuming nonzero inbreeding of unknown parents. J. Dairy Sci. 91:1669-1672. https://doi.org/10 $.3168 /$ jds.2007-0575.

Aguilar, I., and I. Misztal. 2012. INBUPGF90: A program to calculate inbreeding coefficients. Accessed Jan. 7, 2019. http://nce.ads.uga .edu/wiki/doku.php?id=readme.inbupgf90.

Anonymous. 2018. Editorial: A CRISPR definition of genetic modification. 2018. Nat. Plants 4:233. https://doi.org/10.1038/s41477-018 $-0158-1$.

AVMA. 2014. Literature review on the welfare implications of the dehorning and disbudding of cattle. American Veterinary Medicine Association, Schaumburg, IL. Accessed Sep. 20, 2017. https:// www.avma.org/KB/Resources/LiteratureReviews/Pages/Welfare -Implications-of-Dehorning-and-Disbudding-Cattle.aspx.

Bastiaansen, J. W. M., H. Bovenhuis, M. A. M. Groenen, H.-J. Megens, and H. A. Mulder. 2018. The impact of genome editing on the introduction of monogenic traits in livestock. Genet. Sel. Evol. 50:18. https://doi.org/10.1186/s12711-018-0389-7.

Callaway, E. 2018. CRISPR plants now subject to tough GM laws in European Union. Nature 560:16. https://doi.org/10.1038/d41586 -018-05814-6.

Carlson, D. F., C. A. Lancto, B. Zang, E. Kim, M. Walton, D. Oldeschulte, C. Seabury, T. S. Sonstegard, and S. C. Fahrenkrug. 2016. Production of hornless dairy cattle from genome-edited cell lines. Nat. Biotechnol. 34:479-481. https://doi.org/10.1038/nbt.3560.

Carroll, D., A. L. Van Eenennaam, J. F. Taylor, J. Seger, and D. F. Voytas. 2016. Regulate genome-edited products, not genome editing itself. Nat. Biotechnol. 34:477-479. https://doi.org/10.1038/ nbt. 3566 .

Cole, J. B. 2015. A simple strategy for managing many recessive disorders in a dairy cattle breeding program. Genet. Sel. Evol. 47:94. https://doi.org/10.1186/s12711-015-0174-9.

Cole, J. B. 2017a. Management of Mendelian traits in breeding programs by gene editing: A simulation study. bioRxiv. https://doi .org/10.1101/116459 (preprint).

Cole, J. B. 2017b. Python programs and notebooks used for simulation of gene editing in dairy cattle breeding programs. Accessed Sep. 20, 2017. https://github.com/wintermind/gene-editing.

Croney, C., W. Muir, J.-Q. Ni, N. O. Widmar, and G. Varner. 2018. An overview of engineering approaches to improving agricultural animal welfare. J. Agric. Environ. Ethics 31:143-159. https://doi .org/10.1007/s10806-018-9716-9.

Eriksson, S., E. Jonas, L. Rydhmer, and H. Röcklinsberg. 2018. Invited review: Breeding and ethical perspectives on genetically modified and genome edited cattle. J. Dairy Sci. 101:1-17. https://doi .org/10.3168/jds.2017-12962.

FDA. 2017. Guidance for Industry \#187: Regulation of Intentionally Altered Genomic DNA in Animals, Food and Drug Administration, Silver Spring, MD. Accessed Aug. 1, 2018. https://www.fda.gov/ downloads/AnimalVeterinary/GuidanceComplianceEnforcement/ GuidanceforIndustry/UCM113903.pdf. 
Frewer, L. J., G. A. Kleter, M. Brennan, D. Coles, A. R. Fischer, L. M. Houdebine, C. Mora, K. Millar, and B. Salter. 2013. Genetically modified animals from life-science, socio-economic and ethical perspectives: Examining issues in an EU policy context. N. Biotechnol. 30:447-460. https://doi.org/10.1016/j.nbt.2013.03.010.

Goonewardene, L., H. Pang, R. Berg, and M. Price. 1999a. A comparison of reproductive and growth traits of horned and polled cattle in three synthetic beef lines. Can. J. Anim. Sci. 79:123-127. https: //doi.org/10.4141/A98-096.

Goonewardene, L., M. Price, M. Liu, R. Berg, and C. Erichsen. 1999b. A study of growth and carcass traits in dehorned and polled composite bulls. Can. J. Anim. Sci. 79:383-385. https://doi.org/10 .4141/A98-121.

Heller, M. 2018. U.S. agencies clash over who should regulate genetically engineered livestock. Sci. News 19. https://doi.org/10.1126/ science.aat9369.

Kasinathan, P., H. Wei, T. Xiang, J. A. Molina, J. Metzger, D. Broek, S. Kasinathan, D. C. Faber, and M. F. Allan. 2015. Acceleration of genetic gain in cattle by reduction of generation interval. Sci. Rep. 5:8674. https://doi.org/10.1038/srep08674.

Long, C., and K. Gregory. 1978. Inheritance of the horned, scurred, and polled condition in cattle. J. Hered. 69:395-400. https://doi .org/10.1093/oxfordjournals.jhered.a108980.

Maxmen, A. 2017. Gene-edited animals face US regulatory crackdown. NATNEWS 2017. https://doi.org/10.1038/nature.2017.21331.

MCTIC (Ministry of Science, Technology, Innovations and Communications), Brazil. 2018. Technical Opinion 6125/2018. Consultation on the application of Normative Resolution 16 in animal products developed with innovative precision improvement techniques - TIMP. Accessed Jan. 7, 2019. http://sei.mctic.gov.br/verifica .html. Validation code 3509624 and CRC code FFECC97F.

Medugorac, I., D. Seichter, A. Graf, I. Russ, H. Blum, K. H. Göpel, S. Rothammer, M. Förster, and S. Krebs. 2012. Bovine pollednessAn autosomal dominant trait with allelic heterogeneity. PLoS One 7. https://doi.org/10.1371/journal.pone.0039477.

Medugorac, I., A. Graf, C. Grohs, S. Rothammer, Y. Zagdsuren, E. Gladyr, N. Zinovieva, J. Barbieri, D. Seichter, I. Russ, A. Eggen, G. Hellenthal, G. Brem, H. Blum, S. Krebs, and A. Capitan. 2017. Whole-genome analysis of introgressive hybridization and characterization of the bovine legacy of Mongolian yaks. Nat. Genet. 49:470. https://doi.org/10.1038/ng.3775.

Mueller, M. L., J. B. Cole, T. S. Sonstegard, and A. L. Van Eenennaam. 2018a. Simulation of introgression of the POLLED allele into the Holstein breed via conventional breeding versus gene editing. Page 755 in Proc. 11th World Congress on Genetics Applied to Livestock Production., Auckland, New Zealand. Accessed Feb. 25, 2019. http://www.wcgalp.org/proceedings/2018/simulation -introgression-polled-allele-holstein-breed-conventional-breeding -versus.

Mueller, M. L., J. B. Cole, T. S. Sonstegard, and A. L. Van Eenennaam. 2018b. Simulation of introgression of the POLLED allele into the Jersey breed via conventional breeding vs. gene editing. Translational Animal Science 2(Suppl. 1):S57-S60. (Abstr.). https: //doi.org/10.1093/tas/txy054.

NAAB. 2017. Complete List of All Active, Foreign, Genomic and Limited Status Sires (August 2017). Accessed Sep. 15, 2017. https:// www.naab-css.org/database-files.

NAAB. 2018. Complete List of All Active, Foreign, Genomic and Limited Status Sires (March 2018). Accessed Apr. 5, 2017. https:// www.naab-css.org/database-files.

Null, D. J., J. B. Cole, C. Sun, and P. M. Van Raden. 2015. Assignment of polled status using single nucleotide polymorphism genotypes and predicted gene content. J. Dairy Sci. 98(Suppl. 2). https: //m.jtmtg.org/abs/t/63334.
OIE. 2018. Chapter 7.11: Animal welfare and dairy cattle production systems. Terrestrial Animal Health Code. Office International des Epizooties (World Organization for Animal Health), Paris, France.

Pryce, J. E., B. J. Hayes, and M. E. Goddard. 2012. Novel strategies to minimize progeny inbreeding while maximizing genetic gain using genomic information. J. Dairy Sci. 95:377-388. https://doi.org/10 $.3168 /$ jds.2011-4254.

Rothammer, S., A. Capitan, E. Mullaart, D. Seichter, and I. Russ 2014. The $80-\mathrm{kb}$ DNA duplication on BTA1 is the only remaining candidate mutation for the polled phenotype of Friesian origin. Genet. Sel. Evol. 46. https://doi.org/10.1186/1297-9686-46-44.

Rutgers, L. 2003. New Jerseyans' opinions on humane standards for treatment of livestock. Conducted for Farm Sanctuary. Rutgers, NJ: Eagleton Institute of Politics Center for Public Interest Polling. Accessed Sep. 20, 2017. https://www.nj.gov/agriculture/ divisions/ah/pdf/HumaneStandards2010.pdf.

Scheper, C. 2016. Evaluation of breeding strategies for polledness in dairy cattle using a newly developed simulation framework for quantitative and Mendelian traits. Genet. Sel. Evol. 48:50. https:/ /doi.org/10.1186/s12711-016-0228-7.

Schultz-Bergin, M. 2018. Is CRISPR an ethical game changer? J. Agric. Environ. Ethics 31:219-238. https://doi.org/10.1007/s10806 $-018-9721-z$

Spurlock, D. M., M. L. Stock, and J. F. Coetzee. 2014. The impact of 3 strategies for incorporating polled genetics into a dairy cattle breeding program on the overall herd genetic merit. J. Dairy Sci. 97:5265-5274. https://doi.org/10.3168/jds.2013-7746.

Stafford, K. J., and D. J. Mellor. 2011. Addressing the pain associated with disbudding and dehorning in cattle. Appl. Anim. Behav. Sci. 135. https://doi.org/10.1016/j.applanim.2011.10.018.

Tan, W. S., D. F. Carlson, M. W. Walton, S. C. Fahrenkrug, and P. B. Hackett. 2012. Chapter Two - Precision editing of large animal genomes. Adv. Genet. 80:37-97. https://doi.org/10.1016/B978-0 -12-404742-6.00002-8.

Thompson, N. M., N. O. Widmar, M. M. Schutz, J. B. Cole, and C. A. Wolf. 2017. Economic considerations of breeding for polled dairy cows versus dehorning in the United States. J. Dairy Sci. 100:4941-4952. https://doi.org/10.3168/jds.2016-12099.

USDA. 2014. Dairy 2014: Health and Management Practices on U.S. Dairy Operations No. \#696.0218. USDA, Animals and Plant Health Inspection Service, Fort Collins, CO. Accessed Sep. 15 2017. https://www.aphis.usda.gov/animal_health/nahms/dairy/ downloads/dairy14/Dairy14_dr_PartIII.pdf.

Van Eenennaam, A. L. 2018. The importance of a novel product riskbased trigger for gene-editing regulation in food animal species. The CRISPR Journal 1:101-106. https://doi.org/10.1089/crispr .2017 .0023 .

Van Eenennaam, A. L., and A. E. Young. 2018. Public Perception of Animal Biotechnology. Pages 275-303 in: Animal Biotechnology 2. Emerging Breeding Technologies. H. Niemann and C. Wrenzycki, ed. Springer International Publishing, Cham, Switzerland.

Ventura, B. A., M. A. G. von Keyserlingk, and D. M. Weary. 2015. Animal welfare concerns and values of stakeholders within the dairy industry. J. Agric. Environ. Ethics 28:109-126. https://doi.org/10 .1007/s10806-014-9523-x.

Wells, K. D. 2016. History and future of genetically engineered food animal regulation: An open request. J. Transgenic Res. 25:385394. https://doi.org/10.1007/s11248-016-9935-7.

Windig, J. J., R. A. Hoving-Bolink, and R. F. Veerkamp. 2015. Breeding for polledness in Holstein cattle. Livest. Sci. 179. https://doi .org/10.1016/j.livsci.2015.05.021. 